\title{
Comparison of finite element reliability methods
}

\author{
Bruno Sudret ${ }^{\mathrm{a}, *}$, Armen Der Kiureghian ${ }^{\mathrm{b}}$ \\ ${ }^{a} E D F R \& D$, Département Matériaux et Mécanique des Composants, Site des Renardières, Ecuelles, 77818 Moret-sur-Loing Cedex, France \\ ${ }^{\mathrm{b}}$ Department of Civil and Environmental Engineering, University of California, Berkeley, CA 94720, USA
}

\begin{abstract}
The spectral stochastic finite element method (SSFEM) aims at constructing a probabilistic representation of the response of a mechanical system, whose material properties are random fields. The response quantities, e.g. the nodal displacements, are represented by a polynomial series expansion in terms of standard normal random variables. This expansion is usually post-processed to obtain the second-order statistical moments of the response quantities. However, in the literature, the SSFEM has also been suggested as a method for reliability analysis. No careful examination of this potential has been made yet. In this paper, the SSFEM is considered in conjunction with the first-order reliability method (FORM) and with importance sampling for finite element reliability analysis. This approach is compared with the direct coupling of a FORM reliability code and a finite element code. The two procedures are applied to the reliability analysis of the settlement of a foundation lying on a randomly heterogeneous soil layer. The results are used to make a comprehensive comparison of the two methods in terms of their relative accuracies and efficiencies. (C) 2002 Elsevier Science Ltd. All rights reserved.
\end{abstract}

Keywords: Stochastic finite elements; Random fields; Reliability; FORM; Importance sampling

\section{Introduction}

Many applications in mechanics require consideration of stochastic properties of materials, geometry or loads. The basic representation of uncertain parameters in the underlying models is obtained by introducing random variables or fields. Different kinds of analysis accounting for uncertainties can be carried out. Second moment analysis aims at characterizing the second-order statistical moments, i.e. means and variances of response quantities (displacements, strain and stress components, etc.) from those of the input variables. The perturbation method [1-4] and the weighted integral method [5-7] are in this category. On the other hand, reliability methods focus on the calculation of the probability of failure associated with a limit-state function. First-order reliability method (FORM) and SORM approximations and various simulation methods are commonly used in reliability analysis [8]. Because of the typically high level of reliability of civil structures, the failure probability is usually small (in the order of $10^{-2}-10^{-6}$ ).

To account for the spatial variability of uncertain quantities (for instance, that of a material property), a characterization in terms of a random field is usually employed. Through a process of discretization, it is possible

\footnotetext{
* Corresponding author.

E-mail addresses: bruno.sudret@edf.fr (B. Sudret), adk@ce.berkeley.edu (A. Der Kiureghian).
}

to represent the random field by a vector of random variables. One of the methods mentioned above may then be used to carry out second-moment or reliability analysis.

The spectral stochastic finite element method (SSFEM) proposed by Ghanem and Spanos [9] is an approach well suited to analysis involving random fields. It is based on two discretizations of the system of (stochastic) partial differential equations governing the problem under consideration, one in the spatial domain and one in the probabilistic domain. The response (e.g. the random vector of nodal displacements) is cast as a series expansion in standard normal random variables. This can be interpreted as an 'intrinsic' representation of the random response, from which quantities such as statistical moments can be computed by post-processing, either analytically or by simulation.

In the past, SSFEM has been applied to various kinds of problems including two-dimensional elasticity [9], soil mechanics [10], soil dynamics [11], transport in porous media [12] and heat conduction [13]. The first attempt to include material non-linearity such as plasticity can be found in Ref. [14]. In these applications, the authors compute the coefficients of the series expansion of the response, and in most cases post-process the polynomial expression to compute the mean and standard deviation of various response quantities. The possibility of using these probabilistic response expressions for reliability analysis 
has been suggested in Ref. [15], p. 2362 and in Ref. [9], Section 4.1, p. 101-4 and Section 4.4, p. 118-9. However, no careful examination has been made of this potential, although a comparison between FORM/SORM analysis and polynomial chaos expansion for highly non-linear systems has been presented in Ref. [16].

This paper critically examines the applicability of SSFEM in the context of reliability analysis using the FORM approximation as well as importance sampling. The approach is compared with a direct coupling of a FORM reliability code and a finite element code, similar to that used in Refs. [17-19]. The comparison is illustrated through an application to a geotechnical engineering problem involving the settlement of a foundation supported on randomly heterogeneous soil. Specific quantitative measures of accuracy and efficiency of the two methods are given.

\section{Discretization of random fields}

A random field $H(\mathbf{x}, \theta)$ is a collection of random variables associated with a continuous index $\mathbf{x} \in \Omega \subset \mathbb{R}^{n}$, where $\theta \in \Theta$ is the coordinate in the outcome space. With this notation, $H\left(\mathbf{x}, \theta_{0}\right)$ denotes a particular realization of the field, whereas $H\left(\mathbf{x}_{0}, \theta\right)$ is the random variable associated with point $\mathbf{x}_{0}$. Gaussian random fields are of practical interest because they are completely described by a mean function $\mu(\mathbf{x})$, a variance function $\sigma^{2}(\mathbf{x})$ and an autocorrelation coefficient function $\rho\left(\mathbf{x}, \mathbf{x}^{\prime}\right)$.

Discretizing the random field $H(\cdot)$ consists in approximating it by $\hat{H}(\cdot)$, which is defined by means of a finite set of random variables $\left\{\chi_{i}, i=1, \ldots, n\right\}$, grouped in a random vector denoted by $\chi$ :

$H(\mathbf{x}, \theta) \stackrel{\text { Discretization }}{\rightarrow} \hat{H}(\mathbf{x}, \theta)=[\mathscr{F} \mathbf{x}, \boldsymbol{\chi}(\theta)]$

Several methods have been developed in the 1980s to carry out this task, such as the spatial average method [20], the midpoint method [17] and the shape function method [3,4]. A comprehensive review and comparison of these methods is presented in Ref. [21]. These early methods are relatively inefficient, in the sense that a large number of random variables is required to achieve a good approximation of the field. More efficient approaches for discretization of random fields using series expansion methods have been introduced in the past 10 years, including the Karhunen-Loève Expansion (KL) [9], the Orthogonal Series Expansion (OSE) [22] and the Expansion Optimal Linear Estimation (EOLE) method [21]. Reviews of these methods have been presented in Refs. [21,23,24]. As shown in Ref. [24], all three methods result in a representation of the Gaussian field in the form

$\hat{H}(\mathbf{x}, \theta)=\mu(\mathbf{x})+\sum_{i=1}^{M} H_{i}(\mathbf{x}) \xi_{i}(\theta)$ where $\left\{\xi_{i}(\theta), i=1, \ldots, M\right\}$ are standard normal random variables $\left\{H_{i}(\mathbf{x}), i=1, \ldots, M\right\}$ are deterministic functions depending on the correlation structure of the field and $M$ is the number of terms used in the series expansion. The accuracy of the representation depends on $M$ and the particular expansion method used. Details about the respective advantages of each method in terms of accuracy and efficiency can be found in Ref. [24].

\section{Summary of SSFEM}

The SSFEM is detailed in Ref. [9]. For the sake of clarity and completeness, the principles and main steps are presented in the sequel. As mentioned in Section 1, SSFEM is based on two successive discretizations of the system of stochastic partial differential equations governing the problem under consideration:

- A spatial discretization similar to the one used in classical finite element analysis, i.e. involving a mesh of the structure and shape functions.

- A probabilistic discretization of the random field(s) representing the uncertain properties of the constitutive material or loads. The KL expansion has been used in the literature. However, any of the series expansion methods mentioned in Section 2 is equally applicable.

In classical finite element analysis, the basic response quantity is the vector of nodal displacements. In SSFEM, due to the introduction of a random field as an input, the basic response quantity is a random vector of nodal displacements $\mathbf{U}(\theta)$, whose probabilistic content is a priori unknown. Each component $U^{i}(\theta)$ is expanded over the socalled polynomial chaos (see Ref. [24] for a simplified presentation of this concept):

$U^{i}(\theta)=\sum_{j=0}^{P-1} U_{j}^{i} \Psi_{j}\left(\left\{\xi_{k}(\theta)\right\}_{k=1}^{M}\right)$

where $\left\{\xi_{k}(\theta), k=1, \ldots, M\right\}$ denote the standard normal variables appearing in the discretization of the input random field (Eq. (2)), $\Psi_{j}\left(\left\{\xi_{k}(\theta)\right\}_{k=1}^{M}\right)$ are multidimensional Hermite polynomials defined by means of the $\xi_{i}$ s and $P$ is the order of expansion. The latter is connected to the number of random variables $M$ (order of the expansion of the random field) and the highest order $p$ of the polynomials $\Psi_{j}$ by:

$P=\sum_{k=0}^{p}\left(\begin{array}{c}M+k-1 \\ k\end{array}\right)$ (binomial coefficient)

Using classical notation, the finite element method for static problems in linear elasticity eventually yields a linear system of size $N \times N$ ( $N$ being the number of degrees of freedom)

$\mathbf{K} \cdot \mathbf{U}=\mathbf{F}$ 
where $\mathbf{F}$ is the deterministic load vector, and where the global stiffness matrix $\mathbf{K}$ is obtained after assembling the element stiffness matrices $\mathbf{k}^{e}$ :

$\mathbf{K}=\bigcup_{e} \mathbf{k}^{e}, \quad \mathbf{k}^{e}=\int_{\Omega_{e}} \mathbf{B}^{\mathrm{T}} \cdot \mathbf{D} \cdot \mathbf{B} \mathrm{d} \Omega_{e}$

In the above, $\mathbf{D}$ stands for the elasticity matrix and $\mathbf{B}$ is the matrix that relates the components of strain to the element nodal displacements. Suppose that the Young's modulus of the material is a random field. Then the elasticity matrix at point $\mathbf{x}$ can be written as

$\mathbf{D}(\mathbf{x}, \theta)=H(\mathbf{x}, \theta) \mathbf{D}_{0}$

where $\mathbf{D}_{0}$ is a constant deterministic matrix corresponding to a unit value of the Young's modulus. Substituting Eq. (7) in Eq. (6) yields the stochastic stiffness matrix

$\mathbf{K}(\theta)=\bigcup_{e} \mathbf{k}^{e}(\theta)=\bigcup_{e} \int_{\Omega_{e}} \mathbf{B}^{\mathrm{T}} \cdot \mathbf{D}_{0} H(\mathbf{x}, \theta) \cdot \mathbf{B} \mathrm{d} \Omega_{e}$

When the random field $H(\mathbf{x}, \theta)$ appearing in Eq. (8) is discretized as in Eq. (2), the stochastic stiffness matrix takes the form

$\mathbf{K}(\theta) \approx \mathbf{K}_{0}+\sum_{i=1}^{M} \mathbf{K}_{i} \xi_{i}(\theta)$

where the mean- and weighted stiffness matrices $\mathbf{K}_{0}$ and $\mathbf{K}_{i}$ are given by

$\mathbf{K}_{0}=\bigcup_{e} \mathbf{k}_{0}^{e}=\bigcup_{e} \int_{\Omega_{e}} \mu(\mathbf{x}) \mathbf{B}^{\mathrm{T}} \cdot \mathbf{D}_{0} \cdot \mathbf{B} \mathrm{d} \Omega_{e}$

$\mathbf{K}_{i}=\bigcup_{e} \mathbf{k}_{i}^{e}=\bigcup_{e} \int_{\Omega_{e}} H_{i}(\mathbf{x}) \mathbf{B}^{\mathrm{T}} \cdot \mathbf{D}_{0} \cdot \mathbf{B} \mathrm{d} \Omega_{e}$

Collecting the terms in Eq. (3) in a vector form, the random vector of nodal displacements is expanded over the polynomial chaos with $P$ coefficient vectors $\left\{\mathbf{U}_{0}, \ldots\right.$, $\left.\mathbf{U}_{P-1}\right\}$. These coefficients are computed using the Galerkin method for minimizing the residual in the equilibrium equation, leading to:

$\left[\begin{array}{ccc}\mathbf{K}_{00} & \cdots & \mathbf{K}_{0, P-1} \\ \mathbf{K}_{10} & \cdots & \mathbf{K}_{1, P-1} \\ \vdots & & \vdots \\ \mathbf{K}_{P-1,0} & \cdots & \mathbf{K}_{P-1, P-1}\end{array}\right] \cdot\left[\begin{array}{c}\mathbf{U}_{0} \\ \mathbf{U}_{1} \\ \vdots \\ \mathbf{U}_{P-1}\end{array}\right]=\left[\begin{array}{c}\mathbf{F} \\ 0 \\ \vdots \\ 0\end{array}\right]$

where $\left\{\mathbf{K}_{j k}=\sum_{i=0}^{M} c_{i j k} \mathbf{K}_{i}, j, k=0, \ldots, P-1\right\}$ and $c_{i j k}=$ $E\left[\xi_{i} \Psi_{j} \Psi_{k}\right]$ (mathematical expectation of products).

In essence, in SSFEM, the characterization of the random vector of nodal displacements is reduced to solving a linear system of size $(N \times P)$, where $N$ is the number of degrees of freedom of the structure, and $P$ is the order of expansion of the response. In application, $P$ is usually in the range 10-35, which means that the above system of equations can be quite large, even for small size problems. Specific methods for efficiently solving this system of equations have been proposed by Ghanem and Kruger [25].

\section{Finite element reliability analysis}

Reliability methods aim at evaluating the probability of failure of a system whose modeling takes into account randomness. Let $\boldsymbol{\chi}$ denote the set of all basic random variables pertaining to the structure under consideration. If random fields are involved, one of the discretization schemes mentioned earlier can be used to represent them in terms of a finite set of random variables, which are also included in $\boldsymbol{\chi}$. Let $\mathbf{S}$ denote the vector of load effects (displacements, strains, stresses, measures of damage, etc.). These two vectors are related through the mechanical transformation $\mathbf{S}=\mathscr{S}(\chi)$, which in non-trivial cases is defined in an algorithmic sense, e.g. through a finite element computer code. To assess the reliability of the structure, a limit-state function $g$ depending on load effects is defined such that $g(\mathbf{S})>0$ defines the safe state and $g(\mathbf{S}) \leq 0$ defines the failure state. The values of $\mathbf{S}$ satisfying $g(\mathbf{S})=0$ define the limit-state surface of the system. Using this notation, the aim of reliability analysis is to compute the probability of the failure event

$P_{\mathrm{f}}=P(g(\mathscr{S}(\boldsymbol{\chi})) \leq 0)=\int_{g(\mathscr{S}(\boldsymbol{\chi})) \leq 0} f_{\boldsymbol{\chi}}(\boldsymbol{\chi}) \mathrm{d} \boldsymbol{\chi}$

where $f_{\boldsymbol{\chi}}(\boldsymbol{\chi})$ is the joint probability density function (PDF) of the random variables $\boldsymbol{\chi}$. System reliability problems involve a multitude of limit-state functions and a logical expression of events as described above [8].

Direct evaluation of Eq. (13) usually is not possible because of the high dimension of the integral. To overcome this difficulty, the problem is recast in the standard normal space through a probabilistic transformation $\mathscr{Y}(\boldsymbol{X})$ of the basic random variables $\boldsymbol{\chi}$. The Nataf or Rosenblatt transformations can be used for this purpose [8]. The problem then reduces to that of computing

$P_{\mathrm{f}}=\int_{g\left(\mathscr{S} \circ \mathscr{Y}^{-1}(\mathbf{Y})\right)} \varphi(\mathbf{y}) \mathrm{d} \mathbf{y}$

where $\varphi(\mathbf{y})$ denotes the standard normal PDF.

\subsection{First-order reliability method (FORM)}

The FORM consists in replacing the integration domain in Eq. (14) by the half-space obtained from the tangent to the limit-state surface at the design point $\mathbf{y}^{*}$. This is the point satisfying $g\left(\mathscr{S} \circ \mathscr{Y}^{-1}\left(\mathbf{y}^{*}\right)\right)=0$ that is closest to the origin in the standard normal space. The design point $\mathbf{y}^{*}$ is determined using an optimization algorithm, e.g. the improved Hasofer-Lind, Rackwitz-Fiessler (iHLRF) algorithm ([26], see also Refs. [27,28] for other algorithms). The reliability index $\beta$ is then obtained as the norm of $\mathbf{y}^{*}$, from which the FORM approximation of the probability of 


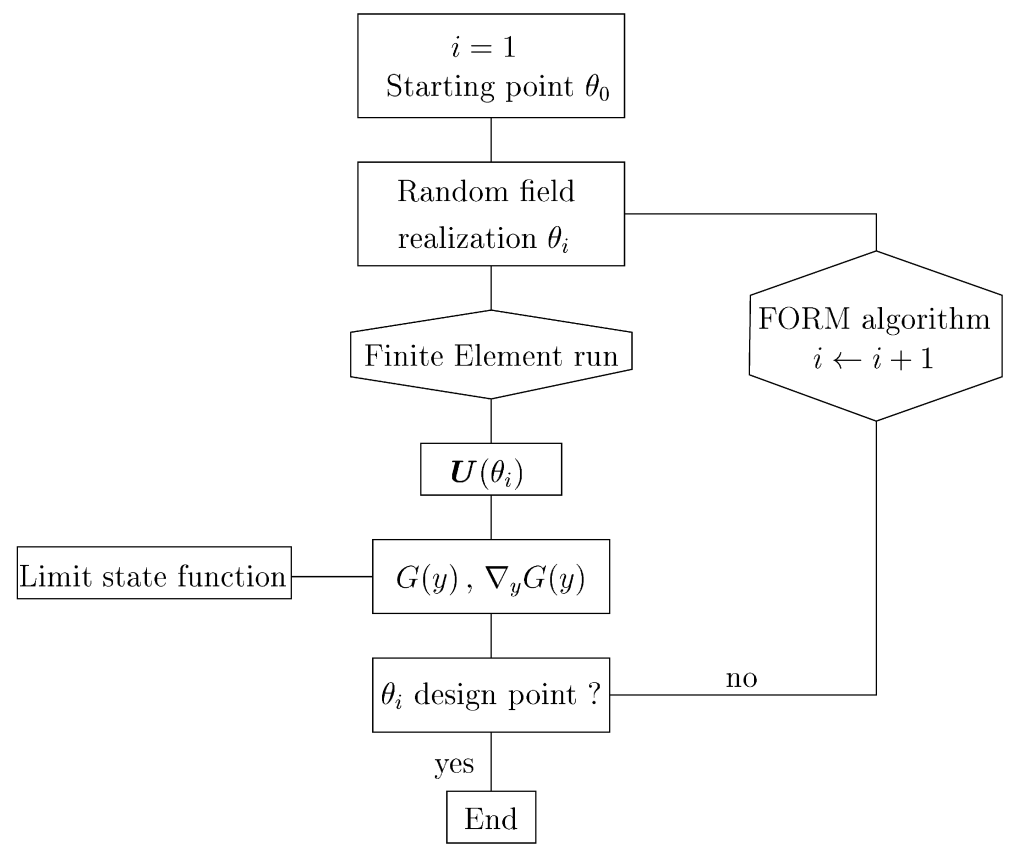

Fig. 1. Direct coupling between a reliability code and a finite element code.

failure is $P_{\mathrm{f}}=\Phi(-\beta)$, where $\Phi(\cdot)$ denotes the standard normal cumulative distribution function $(\mathrm{CDF})$.

The algorithm used to find the design point is an iterative procedure that requires evaluation of the limit-state function $g(\cdot)$ and its gradient with respect to the basic random variables $\boldsymbol{\chi}$ at each step. The former implies computing the mechanical response of the system for a given realization of the basic random variables. The latter involves, through the use of the chain rule of differentiation, the gradient of the mechanical response with respect to the basic random variables $[17,18]$. This can be obtained either from the finite element code itself by use of the direct differentiation method [29], or through a finite difference scheme. In all cases, the reliability code drives the finite element code as shown in Fig. 1.

The direct coupling approach is rather general and can be applied to non-linear and time-variant problems, and to Gaussian as well as non-Gaussian random fields. See Refs. [19,30] for applications to non-linear problems and Ref. [31] for an application in dynamics.

\subsection{Importance sampling}

In order to validate the results obtained by FORM, importance sampling is often used after the FORM analysis (see Ref. [32], Chapter 3). It is based on the following reformulation of Eq. (13):

$$
\begin{aligned}
P_{\mathrm{f}} & =\int_{\mathbb{R}^{n}} I[g(\mathscr{S}(\boldsymbol{\chi})) \leq 0] \frac{f_{\boldsymbol{\chi}}(\boldsymbol{\chi})}{h_{\mathrm{s}}(\boldsymbol{\chi})} h_{\mathrm{s}}(\boldsymbol{\chi}) \mathrm{d} \boldsymbol{\chi} \\
& =E_{h_{\mathrm{s}}}\left[I[g(\mathscr{S}(\boldsymbol{\chi})) \leq 0] \frac{f_{\boldsymbol{\chi}}(\boldsymbol{\chi})}{h_{\mathrm{s}}(\boldsymbol{\chi})}\right]
\end{aligned}
$$

where $n$ is the length of vector $\boldsymbol{\chi}$ and $I[\cdot]$ is the indicator function. Thus the probability of failure is interpreted as the expectation of a quantity with respect to the importance sampling probability density function $h_{\mathrm{s}}(\boldsymbol{\chi})$. It can be estimated as follows:

$P_{\mathrm{f}} \approx \frac{1}{N_{\mathrm{s}}} \sum_{i=1}^{N_{\mathrm{s}}}\left\{I\left[g\left(\mathscr{S}\left(\boldsymbol{\chi}_{i}\right)\right) \leq 0\right] \frac{f_{\boldsymbol{\chi}}\left(\boldsymbol{\chi}_{i}\right)}{h_{\mathrm{s}}\left(\boldsymbol{\chi}_{i}\right)}\right\}$

where $\left\{\boldsymbol{\chi}_{i}, i=1, \ldots, N_{\mathrm{s}}\right\}$ are the sample values. To take advantage from the FORM analysis previously carried out, the sampling PDF is usually centered on the FORM design point, and is assigned a prescribed covariance matrix. By this procedure, the simulation points are concentrated around the design point. Thus, Eq. (16) provides a good estimation of $P_{\mathrm{f}}$ with a relatively small number of samples (e.g. 50-100), regardless of the value of the failure probability. Convergence of the simulation estimate is determined by monitoring the coefficient of variation of the estimate. It is emphasized that the choice of the center of the sampling PDF is arbitrary, meaning that the approach would lead to a correct result even if the design point obtained by FORM were inexact (though in that case the convergence would occur more slowly) or not unique (in this case, the number of samples required for a good accuracy would probably be greater than 50-100).

\section{Reliability analysis by SSFEM}

\subsection{Introduction}

The basic response quantity produced by SSFEM is the 


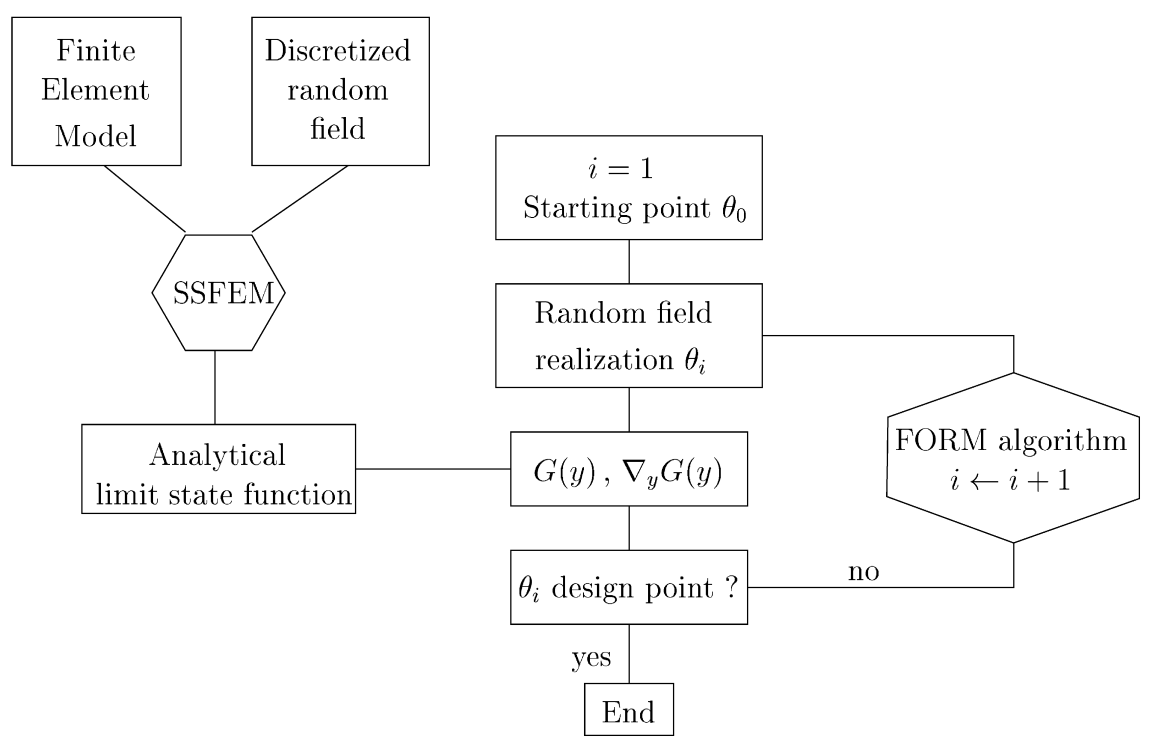

Fig. 2. Post-processing of SSFEM for reliability analysis.

random vector of nodal displacements, cast as an expansion over the polynomial chaos. As in deterministic finite element analysis, quantities such as stress or strain in each Gauss point can be derived in terms of the nodal displacements. Consequently, each response quantity can be cast as a function of standard normal random variables. As an extension, any limit-state function involving these response quantities can be given an analytical expression in terms of standard normal random variables:

$G(\mathbf{S})=G\left(\mathbf{S}\left(\sum_{j=0}^{P-1} U_{j}^{i} \Psi_{j}\left(\left\{\xi_{k}(\theta)\right\}_{k=1}^{M}\right)\right)\right) \equiv G(\boldsymbol{\xi})$

where $G(\xi)$ is the limit-state function in the standard normal space. In the simplest case, if we are interested in the probability that the displacement $U^{i}$ at a particular degree of freedom will exceed the threshold $u_{0}$, then

$G(\boldsymbol{\xi})=u_{0}-\sum_{j=0}^{P-1} U_{j}^{i} \Psi_{j}\left(\left\{\xi_{k}(\theta)\right\}_{k=1}^{M}\right)$

the coefficients $U_{j}^{i}$ being computed by the SSFEM procedure described in Section 3.

\subsection{Post-processing of SSFEM for reliability analysis}

Once SSFEM is carried out and the coefficients in the polynomial expansion of the response are computed, any desired probabilistic analysis can be performed with the approximate limit-state function. In particular, following Section 4.1, a FORM analysis can be carried out to approximately determine the probability of failure associated with the limit-state function (17). It is emphasized that this limit-state function is analytical and already cast in the standard normal space. Its evaluation is possible at a low cost, since no additional finite element calculations are needed once the SSFEM analysis has been carried out. Its gradient with respect to the random variables $\xi_{k}$ is also known analytically and, thus, easy to compute. A chart describing the procedure is given in Fig. 2.

Moreover, it is easy to improve the FORM estimate of the probability of failure by importance sampling around the design point, as described in Section 4.2.

Additional evaluations of the limit-state function required for that purpose are computationally inexpensive. The direct Monte Carlo simulation can also be used efficiently, if the probability of failure is not too small.

Finally, it is possible to obtain at low cost the PDF of any response quantity by sensitivity analysis. From Eqs. (13) and (18), one has

$P_{\mathrm{f}}=P\left(u_{0} \leq U^{i}\right)=1-F_{i}\left(u_{0}\right)$

where $F_{i}$ is the cumulative distribution function of random variable $U^{i}$. By differentiation, the PDF of $U^{i}$ is

$f_{i}\left(u_{0}\right)=\frac{\mathrm{d} F_{i}}{\mathrm{~d} u_{0}}=-\frac{\mathrm{d} P_{\mathrm{f}}}{\mathrm{d} u_{0}}=\varphi\left(\beta\left(u_{0}\right)\right) \frac{\mathrm{d} \beta}{\mathrm{d} u_{0}}$

Classical FORM sensitivity analysis [8] allows to compute the derivative of the reliability index $\beta$ with respect to the parameter $u_{0}$ by

$\frac{\mathrm{d} \beta}{\mathrm{d} u_{0}}=\frac{1}{\left\|\boldsymbol{\nabla}_{\xi} g\left(\mathbf{U}\left(\boldsymbol{\xi}^{*}\left(u_{0}\right)\right), u_{0}\right)\right\|} \frac{\partial g\left(\boldsymbol{\xi}, u_{0}\right)}{\partial u_{0}}$

In this expression, the derivative of $g$ with respect to $u_{0}$ is simply 1 due to Eq. (18). Thus, substituting Eq. (21) in Eq. (20) yields

$f_{i}\left(u_{0}\right)=\frac{\varphi\left(\beta\left(u_{0}\right)\right.}{\left\|\nabla_{\xi} g\left(\mathbf{U}\left(\boldsymbol{\xi}^{*}\left(u_{0}\right)\right), u_{0}\right)\right\|}$

To compute the entire PDF of a nodal displacement, a 


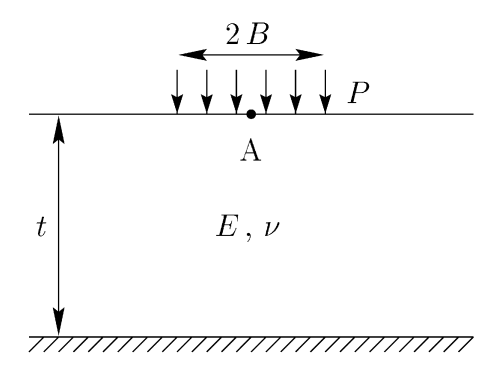

Fig. 3. Settlement of a foundation-problem definition.

FORM analysis is carried out for different thresholds $u_{0}$, yielding $\beta\left(u_{0}\right)$ and $\boldsymbol{\xi}^{*}\left(u_{0}\right)$. Eq. (22) is then evaluated for each $u_{0}$.

The accuracy achieved in the above analysis with SSFEM will depend on the accuracy of the representation of the limit-state function in terms of the polynomially expanded response quantities. This is examined in detail in Section 6.

\section{Application: foundation on a heterogeneous soil layer}

\subsection{Implementation of the various approaches}

The comparison of the methods described in Sections 4 and 5 requires:

- A software for discretizing random fields.

- A reliability software allowing to determine the design point for FORM analysis and to perform simulation, importance sampling, etc.

- A deterministic finite element code capable of considering space-variant material properties (i.e. realizations of a random field).

- An implementation of SSFEM.

For the second item, Finite Element Reliability Using Matlab (FERUM) was used. This is an educational and research software developed at the University of California at Berkeley by Haukaas and Der Kiureghian [33]. For the other three items, softwares were developed in the Matlab environment as well. These include a 'random field toolbox' implementing the various series expansion methods mentioned in Section 2 as well as a 'SSFEM toolbox'. Details about the implementation are given in Ref. [24] and

Table 1

Parameters of the deterministic model

\begin{tabular}{lll}
\hline Parameter & Symbol & Value \\
\hline Soil layer thickness & $T$ & $30 \mathrm{~m}$ \\
Foundation width & $2 B$ & $10 \mathrm{~m}$ \\
Applied pressure & $P$ & $0.2 \mathrm{MPa}$ \\
Soil Young's modulus & $E$ & $50 \mathrm{MPa}$ \\
Soil Poisson's ratio & $\nu$ & 0.3 \\
Mesh width & $L$ & $60 \mathrm{~m}$ \\
\hline
\end{tabular}

download files can be found at http://www.ce.berkeley. edu/ haukaas under the item 'FERUM'. For the direct coupling approach, the direct differentiation method is used to compute the gradient of the finite element response, see details in Ref. [24].

\subsection{Description of the deterministic problem}

Consider an elastic soil layer of thickness $t$ lying on a rigid substratum. A superstructure to be founded on this soil mass is idealized as a uniform pressure $P$ applied over a length $2 B$ of the free surface (Fig. 3). The soil is modeled as an elastic linear isotropic material. A plane strain analysis is carried out.

Due to the symmetry, half of the structure is modeled by finite elements. Strictly speaking, there is no symmetry in the system when random fields of material properties are introduced. However, it is believed that this simplification does not significantly influence the results. The parameters selected for the deterministic model are listed in Table 1.

A refined mesh was first used to obtain the 'exact' maximum displacement under the foundation (point $\mathrm{A}$ in Fig. 3). Less refined meshes were then tried in order to design a mesh with as few elements as possible that yielded no more than $1 \%$ error in the computed maximum settlement. The mesh shown in Fig. 4a was eventually chosen. It contains 99 nodes and 80 elements.

For the input parameters given in Table 1, the maximum displacement obtained with the most refined mesh is $u_{\mathrm{A}}^{\text {exact }}=5.49 \mathrm{~cm}$. The value obtained with the mesh in Fig. 4a is $u_{\mathrm{A}}^{0}=5.42 \mathrm{~cm}$. The deformed shape is plotted in Fig. 4b.

\subsection{Description of the probabilistic data}

The assessment of the serviceability of the foundation described in the above paragraph is now investigated under the assumption that the Young's modulus of soil is a homogeneous random field. The limit-state function is defined in terms of the maximum settlement $u_{\mathrm{A}}$ at the center of the foundation:

$g\left(\mathbf{U}(\boldsymbol{\xi})=u_{0}-u_{\mathrm{A}}(\boldsymbol{\xi})\right.$

where $u_{0}$ is an admissible threshold initially set equal to $10 \mathrm{~cm}$.

The Young's modulus of the soil is considered to vary only in the vertical direction, so that it is modeled as a onedimensional random field along the depth. This is a reasonable model for a layered soil medium. For the sake of simplicity, the field is assumed to be Gaussian and homogeneous. Initially, its second-moment properties are considered to be the mean $\mu_{\mathrm{E}}=50 \mathrm{MPa}$, the coefficient of variation $\delta_{\mathrm{E}}=\sigma_{\mathrm{E}} / \mu_{\mathrm{E}}=0.2$, and the autocorrelation coefficient function $\rho_{\mathrm{EE}}\left(z, z^{\prime}\right)=\exp \left(-\left|z-z^{\prime}\right| / \ell\right)$, where $z$ is the depth coordinate and $\ell=30 \mathrm{~m}$ is the correlation length.

The accuracy of the random field discretization depends 


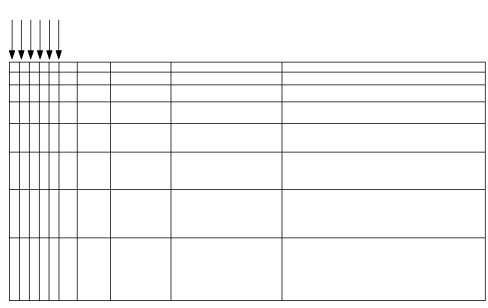

a - Mesh

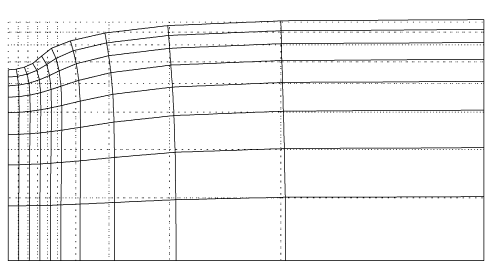

b - Deformed shape

Fig. 4. Finite element mesh and deformed shape for mean values of the parameters by a deterministic analysis: (a) mesh; (b) deformed shape.

on the number of terms $M$ retained in the expansion. For each value of $M$, a global indicator of the accuracy of the discretization, $\overline{\boldsymbol{\varepsilon}}$, is computed from

$\overline{\boldsymbol{\varepsilon}}=\frac{1}{|\Omega|} \int_{\Omega} \boldsymbol{\varepsilon r r}(\mathbf{x}) \mathrm{d} \Omega$

where the point-wise error estimator $\boldsymbol{\varepsilon r r}(\mathbf{x})$ is defined as:

$\boldsymbol{\varepsilon} \operatorname{rr}(\mathbf{x})=\frac{\operatorname{Var}[H(\mathbf{x})-\hat{H}(\mathbf{x})]}{\operatorname{Var}[H(\mathbf{x})]}$

Table 2 lists the error estimates for varying $M$. It appears that $M=3$ is sufficient to assure less than $10 \%$ error in the discretization. Of course this value is closely related to the parameters defining the random field, particularly the correlation length $\ell$.

\subsection{Comparison between direct coupling and SSFEM post- processing}

Using the direct coupling approach, the reliability index $\beta_{\text {direct }}$ is computed for different orders of expansion $M$ of the input random field. Results are reported in Table 3, column \#2. For the initial choice of the parameters, it appears that the direct coupling provides two-digit accuracy in the reliability index $\beta_{\text {direct }}$ for $M \geq 2$, which approximately corresponds to a discretization error $\overline{\boldsymbol{\varepsilon}} \approx 10 \%$.

When using SSFEM, the highest order $p$ of polynomials included in the series expansion of the response must also be specified. For each value of $M$ and $p$, the corresponding number of terms in this expansion, $P$, is given in Table 3 , column \#4. Following the method described in Section 5.2, the reliability index is obtained by FORM post-processing of the SSFEM result, and is given in column \#5.

As expected, $\beta_{\text {SSFEM }}$ converges to $\beta_{\text {direct }}$ for each value of $M$, when the order $p$ of the polynomial chaos expansion is

Table 2

Accuracy of the random field discretization

\begin{tabular}{ll}
\hline Order of expansion $M$ & Error estimator $\bar{\varepsilon}$ \\
\hline 1 & 0.269 \\
2 & 0.129 \\
3 & 0.082 \\
4 & 0.060 \\
5 & 0.048
\end{tabular}

increased (results should be identical for $p=\infty$ ). From the results in Table 3, it appears that at least $p=3$ should be selected to have $5 \%$ accuracy in the reliability index.

\subsection{Parametric study}

A comprehensive comparison of the two approaches is presented by Sudret and Der Kiureghian [24], where the influences of various parameters are investigated. The main results are reported in the sequel.

\subsubsection{Influence of the reliability index}

The threshold $u_{0}$ in the limit-state function is varied in this section from 6 to $30 \mathrm{~cm}$, leading to an increasing reliability index. $M=2$ is used. The results are reported in Table 4.

Using the direct coupling approach, it is observed that the number of iterations required to determine the design point (always starting from the mean point) increases with $\beta$ (column \#3 in Table 4). However, convergence is achieved regardless of the value of the threshold. The accuracy of the results obtained with $M=2$ (compared to those obtained with $M=3$, not presented in the table) is the same regardless of the value of $u_{0}$. In other words, for a given discretized random field, this approach provides correct

Table 3

Reliability index $\beta$-influence of the orders of expansion $M$ and $p$

\begin{tabular}{|c|c|c|c|c|}
\hline$M$ & $\beta_{\text {direct }}$ & $p$ & $P$ & $\beta_{\text {SSFEM }}$ \\
\hline \multirow[t]{5}{*}{1} & 2.694 & 1 & 2 & 4.665 \\
\hline & & 2 & 3 & 3.008 \\
\hline & & 3 & 4 & 2.741 \\
\hline & & 4 & 5 & 2.685 \\
\hline & & 5 & 6 & 2.681 \\
\hline \multirow[t]{5}{*}{2} & 2.631 & 1 & 3 & 4.510 \\
\hline & & 2 & 6 & 2.904 \\
\hline & & 3 & 10 & 2.656 \\
\hline & & 4 & 15 & 2.611 \\
\hline & & 5 & 21 & 2.614 \\
\hline \multirow[t]{3}{*}{3} & 2.627 & 1 & 4 & 4.487 \\
\hline & & 2 & 10 & 2.885 \\
\hline & & 3 & 20 & 2.645 \\
\hline \multirow[t]{2}{*}{4} & 2.627 & 1 & 5 & 4.480 \\
\hline & & 2 & 15 & 2.885 \\
\hline
\end{tabular}


Table 4

Influence of the threshold in the limit-state function

\begin{tabular}{|c|c|c|c|c|c|}
\hline$u_{0}(\mathrm{~cm})$ & $\beta_{\text {direct }}$ & \# Iterations & $p$ & $P$ & $\beta_{\text {SSFEM }}$ \\
\hline \multirow[t]{5}{*}{6} & 0.553 & 4 & 1 & 3 & 0.392 \\
\hline & & & 2 & 6 & 0.504 \\
\hline & & & 3 & 10 & 0.564 \\
\hline & & & 4 & 15 & 0.564 \\
\hline & & & 5 & 21 & 0.552 \\
\hline \multirow[t]{5}{*}{8} & 1.856 & 6 & 1 & 3 & 2.451 \\
\hline & & & 2 & 6 & 1.859 \\
\hline & & & 3 & 10 & 1.821 \\
\hline & & & 4 & 15 & 1.842 \\
\hline & & & 5 & 21 & 1.858 \\
\hline \multirow[t]{5}{*}{10} & 2.631 & 7 & 1 & 3 & 4.509 \\
\hline & & & 2 & 6 & 2.904 \\
\hline & & & 3 & 10 & 2.655 \\
\hline & & & 4 & 15 & 2.610 \\
\hline & & & 5 & 21 & 2.614 \\
\hline \multirow[t]{5}{*}{12} & 3.143 & 7 & 1 & 3 & 6.568 \\
\hline & & & 2 & 6 & 3.787 \\
\hline & & & 3 & 10 & 3.298 \\
\hline & & & 4 & 15 & 3.161 \\
\hline & & & 5 & 21 & 3.126 \\
\hline \multirow[t]{5}{*}{15} & 3.648 & 10 & 1 & 3 & 9.656 \\
\hline & & & 2 & 6 & 4.926 \\
\hline & & & 3 & 10 & 4.065 \\
\hline & & & 4 & 15 & 3.782 \\
\hline & & & 5 & 21 & 3.674 \\
\hline \multirow[t]{5}{*}{20} & 4.139 & 12 & 1 & 3 & 14.803 \\
\hline & & & 2 & 6 & 6.523 \\
\hline & & & 3 & 10 & 5.054 \\
\hline & & & 4 & 15 & 4.533 \\
\hline & & & 5 & 21 & 4.304 \\
\hline \multirow[t]{5}{*}{30} & 4.601 & 13 & 1 & 3 & 25.096 \\
\hline & & & 2 & 6 & 9.093 \\
\hline & & & 3 & 10 & 6.498 \\
\hline & & & 4 & 15 & 5.564 \\
\hline & & & 5 & 21 & 5.118 \\
\hline
\end{tabular}

results for the reliability index regardless of the level of the reliability.

When using SSFEM up to order $p=5$, it appears that fair results (i.e. less than $5 \%$ discrepancy between $\beta_{\text {SSFEM }}$ and $\beta_{\text {direct }}$ ) are obtained only for $u_{0} \leq 20 \mathrm{~cm}$. It is emphasized that the present example was designed such that only two terms $(M=2)$ provide sufficient accuracy in the discretization of the random field, thus allowing the use of up to fifthorder polynomial chaos terms in the expansion of the response. In practice, the number of terms necessary to achieve an accurate discretization of the random field is usually larger than 2 . As a result, only second- or third-order polynomial chaos terms ( $p=2$ or 3 ) in the SSFEM response expansion are practically applicable. Restricting the analysis to the third-order SSFEM, it is seen from Table 4 that the SSFEM estimation of the reliability index is fair only if this index is less than or equal to 3 . It is evident that higher-order terms in the SSFEM
Table 5

Results for shorter correlation length $(\ell=10 \mathrm{~m})$

\begin{tabular}{|c|c|c|c|c|c|}
\hline$M$ & $\overline{\boldsymbol{\varepsilon}}$ & $\beta_{\text {direct }}$ & $p$ & $P$ & $\beta_{\text {SSFEM }}$ \\
\hline \multirow[t]{5}{*}{1} & 0.550 & 3.441 & 1 & 3 & 6.198 \\
\hline & & & 2 & 6 & 3.978 \\
\hline & & & 3 & 10 & 3.583 \\
\hline & & & 4 & 15 & 3.474 \\
\hline & & & 5 & 21 & 3.443 \\
\hline \multirow[t]{5}{*}{2} & 0.335 & 3.215 & 1 & 3 & 5.778 \\
\hline & & & 2 & 6 & 3.690 \\
\hline & & & 3 & 10 & 3.327 \\
\hline & & & 4 & 15 & 3.232 \\
\hline & & & 5 & 21 & 3.208 \\
\hline \multirow[t]{3}{*}{3} & 0.232 & 3.181 & 1 & 3 & 5.671 \\
\hline & & & 2 & 6 & 3.625 \\
\hline & & & 3 & 10 & 3.277 \\
\hline \multirow[t]{2}{*}{4} & 0.175 & 3.180 & 1 & 3 & 5.646 \\
\hline & & & 2 & 6 & 3.608 \\
\hline 5 & 0.140 & 3.179 & - & - & - \\
\hline 10 & 0.071 & 3.179 & - & - & - \\
\hline
\end{tabular}

expansion are necessary in order to accurately compute the far tail of the distribution of $u_{\mathrm{A}}$.

\subsubsection{Influence of the correlation length}

To investigate the influence of the correlation length, $\ell=10 \mathrm{~m}$ is considered (instead of $30 \mathrm{~m}$ ), while keeping the initial values of all remaining parameters. The results are reported in Table 5 .

Comparing column \#2 of Table 5 with column \#2 of Table 2 (the latter corresponding to $\ell=30 \mathrm{~m}$ ), it is evident that a larger number of terms $M$ is now required in order to achieve an acceptable accuracy in the discretization of the random field. However, for $M \geq 3$, i.e. $\overline{\boldsymbol{\varepsilon}} \leq 23 \%$, a twodigit accuracy in the reliability index is achieved when the direct coupling method is used.

When using SSFEM, it appears that fair results (i.e. less than $5 \%$ discrepancy between $\beta_{\text {SSFEM }}$ and $\beta_{\text {direct }}$ ) are obtained for $M \geq 2-3$, and $p \geq 3$. Higher orders of the polynomial chaos expansion would lead to an enormous amount of computation time.

As a conclusion, the direct coupling approach is applicable regardless of the correlation length of the field, since it is computationally inexpensive even for $M=10$ or more. In contrast, due to the computation cost, it would not be practical to use the SSFEM with $p>2$ when $M=10$ or more. The computed reliability index with a lower order expansion may not be sufficiently accurate.

\subsubsection{Influence of the coefficient of variation}

In this section, the order of expansion $M$ is set equal to 2 and the threshold in the limit-state function is $u_{0}=20 \mathrm{~cm}$. The reliability index is computed for different coefficients of 
Table 6

Influence of the coefficient of variation of the input random field

\begin{tabular}{|c|c|c|c|c|}
\hline$\delta_{\mathrm{E}}$ & $\beta_{\text {direct }}$ & $p$ & $P$ & $\beta_{\text {SSFEM }}$ \\
\hline \multirow[t]{5}{*}{0.1} & 8.277 & 1 & 3 & 30.706 \\
\hline & & 2 & 6 & 13.769 \\
\hline & & 3 & 10 & 10.702 \\
\hline & & 4 & 15 & 9.578 \\
\hline & & 5 & 21 & 9.043 \\
\hline \multirow[t]{5}{*}{0.2} & 4.132 & 1 & 3 & 14.803 \\
\hline & & 2 & 6 & 6.523 \\
\hline & & 3 & 10 & 5.054 \\
\hline & & 4 & 15 & 4.535 \\
\hline & & 5 & 21 & 4.303 \\
\hline \multirow[t]{5}{*}{0.3} & 2.759 & 1 & 3 & 9.257 \\
\hline & & 2 & 6 & 3.925 \\
\hline & & 3 & 10 & 2.994 \\
\hline & & 4 & 15 & 2.666 \\
\hline & & 5 & 21 & 2.467 \\
\hline \multirow[t]{5}{*}{0.4} & 2.069 & 1 & 3 & 6.301 \\
\hline & & 2 & 6 & 2.455 \\
\hline & & 3 & 10 & 1.708 \\
\hline & & 4 & 15 & $0.807^{\mathrm{a}}$ \\
\hline & & 5 & 21 & $2.045^{\mathrm{a}}$ \\
\hline \multirow[t]{5}{*}{0.5} & 1.655 & 1 & 3 & 4.380 \\
\hline & & 2 & 6 & 1.370 \\
\hline & & 3 & 10 & 3.062 \\
\hline & & 4 & 15 & $1.592^{\mathrm{a}}$ \\
\hline & & 5 & 21 & 1.227 \\
\hline
\end{tabular}

${ }^{a}$ For these values, the iHLRF algorithm applied after SSFEM has not converged after 30 iterations.

Table 7

Computer processing time required by direct coupling and SSFEM methods

\begin{tabular}{|c|c|c|c|c|}
\hline$M$ & $\mathrm{CPT}^{\mathrm{a}}$ direct coupling $\left(^{\prime \prime}\right)$ & $p$ & $P$ & $\mathrm{CPT}^{\mathrm{a}}$ SSFEM $\left(^{\prime \prime}\right)$ \\
\hline \multirow[t]{5}{*}{1} & 20.6 & 1 & 2 & 2.3 \\
\hline & & 2 & 3 & 3 \\
\hline & & 3 & 4 & 3.2 \\
\hline & & 4 & 5 & 4.0 \\
\hline & & 5 & 6 & 4.8 \\
\hline \multirow[t]{5}{*}{2} & 33.6 & 1 & 3 & 3.9 \\
\hline & & 2 & 6 & 8.0 \\
\hline & & 3 & 10 & 22.6 \\
\hline & & 4 & 15 & 58.2 \\
\hline & & 5 & 21 & 129.0 \\
\hline \multirow[t]{4}{*}{3} & 43.7 & 1 & 4 & 4.7 \\
\hline & & 2 & 10 & 30.3 \\
\hline & & 3 & 20 & 296.4 \\
\hline & & 4 & 35 & 1888.7 \\
\hline \multirow[t]{2}{*}{4} & 53.8 & 1 & 5 & 8.7 \\
\hline & & 2 & 15 & 127.4 \\
\hline 5 & 65.8 & 1 & 6 & 11.4 \\
\hline
\end{tabular}

a The CPT for a deterministic finite element run with constant Young's modulus is $0.57^{\prime \prime}$. variation $\delta_{\mathrm{E}}$ of the input random field. Results are reported in Table 6.

When the direct coupling is used, convergence of the FORM algorithm is always achieved, the number of required iterations varying from 4 to 12 , depending on the level of $\beta$ (more iterations required for larger $\beta$ ). The values obtained are within $1 \%$ of those obtained with $M=3$ (not presented here). It is observed that the reliability index rapidly decreases with increasing variability in the Young's modulus.

When SSFEM is used, poor results are obtained for $\delta_{\mathrm{E}}=$ 0.1 . This is expected because this value corresponds to a large value of the reliability index, for which the SSFEM approach does not provide accurate results (see above for the influence of the reliability index). For larger $\delta_{\mathrm{E}}$, the results are not good either. In some cases the FORM analysis after SSFEM does not converge, and in other cases it converges to a wrong design point, especially when the order of the polynomial chaos is large. This may be explained by the fact that the polynomial response surface associated with SSFEM is undulatory in this case (due to higher order polynomials) and may have several local design points. As an example, for the case of $\delta_{\mathrm{E}}=0.4$, it is observed that the convergence to the true reliability index is not monotonic with increasing $p$. Thus, the result obtained with SSFEM for a given expansion order cannot be a priori positioned with respect to the true value of the reliability index.

From these examples, it appears that SSFEM coupled with FORM cannot be applied safely for large coefficients of variation of the input (e.g. $\delta_{\mathrm{E}}>0.3$ ), whereas the results obtained for the reliability index by the direct coupling method are reliable regardless of the value of $\delta_{\mathrm{E}}$.

\subsection{Comparison of efficiency}

The computer processing time (CPT) required by the direct coupling approach and by SSFEM and its postprocessing are reported in Table 7 . These are for the initial values of the parameters. The bold characters correspond to choices of the parameters $(M, p)$ that give fair estimates of the reliability index.

From column \#2 of Table 7 , it is seen that the CPT required by the direct coupling method is linearly increasing with the order of the expansion $M$. This can be explained as follows: the only step that is modified in the finite element analysis when $M$ is changed is the computation of the element stiffness matrices. Each of these matrices requires evaluation of the realization of the random field at the element Gauss points, and each evaluation takes a time exactly proportional to the order of the expansion $M$ (Eq. (2)). The number of the gradients computed is also proportional to $M$.

In contrast, when using SSFEM, the CPT increases extremely rapidly with the order of the polynomial chaos expansion. Thus, the method can be applied efficiently only 
Table 8

Post-processing of the SSFEM results-comparison between FORM and importance sampling

\begin{tabular}{llll}
\hline$M$ & $p$ & $\beta_{\text {SSFEM }}^{\text {FORM }}$ & $\beta_{\text {SSFEM }}^{\text {IS }}$ \\
\hline 1 & 1 & 4.665 & 4.669 \\
& 2 & 3.008 & 3.012 \\
& 3 & 2.741 & 2.738 \\
& 4 & 2.685 & 2.689 \\
& 1 & 4.510 & 4.515 \\
& 2 & 2.904 & 2.891 \\
& 3 & 2.656 & 2.633 \\
& 4 & 2.611 & 2.578 \\
& 5 & 2.614 & 2.580 \\
& 1 & 4.487 & 4.490 \\
& 2 & 2.889 & 2.872 \\
& 3 & 2.645 & 2.608 \\
\hline
\end{tabular}

when a small number of terms $M$ allows to describe the random field accurately, and when the reliability index under consideration is sufficiently small so that the second or third-order SSFEM gives a fairly accurate estimate.

\subsection{Application of importance sampling}

So far, the SSFEM result has been post-processed by FORM to obtain a first-order approximation of the probability of failure of the system. It could be argued that this is not the best way of post-processing the SSFEM result for reliability analysis, since:

- the analytical polynomial expression of the limit-state function contains information that is lost when the FORM linearization is employed,

- the limit-state surface obtained from SSFEM could be globally accurate, but not around the design point, such that applying FORM could give poor results.

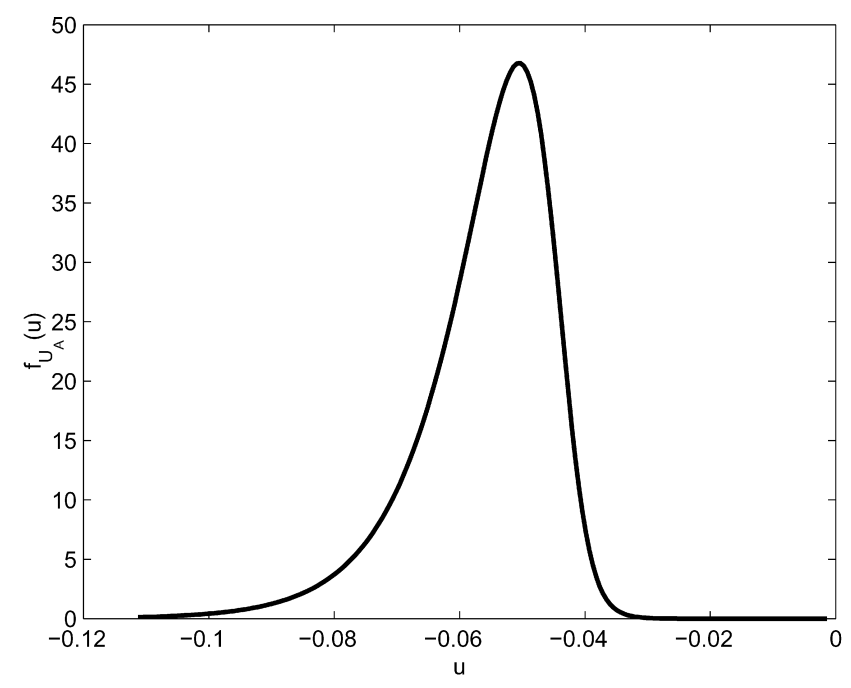

Fig. 5. Probability density function of the maximum displacement obtained by multiple FORM analyses after SSFEM.
To determine whether the FORM approximation deteriorates the SSFEM result, importance sampling around the design point [32] is used after the FORM analysis based on the limit-state function (23), see Section 4.2. This kind of analysis with SSFEM is inexpensive, because the limit-state function is cast in an analytical form. The sampling probability density function used in the analysis is Gaussian with a unit standard deviation and centered at the design point. Ten thousand samples are used for each case. The estimated probability of failure is then transformed back into the reliability index $\beta_{\mathrm{SSFEM}}^{\mathrm{IS}}$ for comparison purposes. The results are summarized in Table 8 .

The FORM is exact for $M=1$ regardless of the value of $p$ (because the limit-state surface is reduced to a single point), and when $p=1$ regardless of $M$ (because the limitstate surface is then an hyperplane). For these cases, it can be seen in Table 8 that importance sampling gives exactly the same results as FORM, the last-digit discrepancy being due to the finite sample size.

Significant discrepancies between the two approaches appear only for higher orders of polynomial chaos expansion, e.g. $p \geq 3$. In any case, they do not exceed $2 \%$ of the value of the reliability index, which means that the FORM approximation is satisfactory in all cases. Thus, it can be concluded that, for the present example, the limitstate surface defined analytically by the SSFEM analysis is sufficiently smooth so that FORM gives good results in comparison to importance sampling. It follows that errors observed in the reliability estimates based on SSFEM in the previous sections are due to the truncation of the polynomial chaos expansions and not due to the FORM approximation.

\subsection{Probability density function of a response quantity}

As mentioned earlier, an important advantage of SSFEM is the availability of an analytical form for the response quantities. After the coefficients in the SSFEM expansion are obtained, reliability analysis for any limit-state function can be easily carried out without additional finite element calculations. For example, one can easily compute the probability density function of a response quantity, as described in Section 5.2.

As an example, the PDF of the nodal displacement $U_{\mathrm{A}}$ is plotted in Fig. 5. 200 points are used, i.e. 200 reliability problems are solved. This is done in a matter of seconds on a personal computer. To improve the efficiency, the starting point of each analysis is chosen as the design point of the previous analysis. This allows convergence of the iHLRF algorithm within three iterations. Of course, similar analysis can be carried out by the direct coupling approach. However, in that case, a few additional finite element calculations at each threshold are necessary.

It can be seen that the obtained PDF has its mode close to $u_{0}=-5.42 \mathrm{~cm}$, which is the value obtained from the deterministic finite element analysis. It should be emphasized that, whereas the central region of this distribution can 
be expected to be accurate, the far tails may be inaccurate, since they correspond to high reliability indices that are not computed accurately by the SSFEM.

\section{Conclusions}

Two methods for finite element reliability analysis involving random fields are reviewed and compared. One method is based on a direct coupling of finite element and reliability codes, and the other involves post-processing of results obtained from the SSFEM. The paper critically evaluates the purported potential of SSFEM for reliability analysis. The comparison is carried out for an example application involving the settlement of a foundation on randomly heterogeneous soil. While deriving general conclusions from a single numerical example is always dangerous, the comparison provides ample evidence regarding the scope and limitations of SSFEM for reliability analysis.

As far as the direct coupling method is concerned, the approach appears to be robust and efficient for the example problem, regardless of the parameter values. The cost of the analysis increases linearly with the order of the expansion of the input random field.

With SSFEM, fair results for the reliability index can be obtained only with high order polynomial chaos expansions $(p \geq 3)$. However, when more than two or three terms are used in the discretization of the random field, the computational time with a high-order polynomial chaos expansion rapidly increases and becomes prohibitive. Consequently, only results obtained with a low order polynomial chaos expansion are practically available in such cases. They appear poor compared to those obtained by direct coupling. In some cases, the computed reliability index can be in gross error. For the example application, this occurs when the input coefficient of variation is large or when the reliability index is large.

The case of $\log$-normal input random fields was also investigated in Ref. [24], using the discretization scheme proposed in Ref. [34]. The conclusions are in essence similar to those presented in this paper. However the computation cost for a given choice of $(M, p)$ is even greater than in the Gaussian case. This is due to the fact that the input random field is discretized using the polynomial chaos as well, which requires more terms than the series expansion used in the Gaussian case.

As an overall conclusion, it is found that SSFEM has limited applicability to reliability problems involving small failure probabilities. The polynomial chaos expansion provides a global fit to each response quantity, which may be good in the central region of the respective distribution, but poor in the tail regions. Since small-probability events are influenced by the tail regions of these probability distributions, accurate results from SSFEM cannot be expected for such problems. This limitation is more severe for problems involving random fields with short correlation lengths or large coefficients of variation.

The above conclusions do not invalidate the effectiveness of SSFEM for second-moment analysis. Furthermore, SSFEM might be effective in providing a good approximation to the central region of the probability density function of a response quantity.

\section{Acknowledgements}

This work has been carried out during the post-doctoral stay of the first author at the University of California at Berkeley. This stay was supported by Ecole Nationale des Ponts et Chaussées (France) and by Electricité de France under Award Number D56395-T6L29-RNE861. These supports are gratefully acknowledged.

\section{References}

[1] Handa K, Andersson K. Application of finite element methods in the statistical analysis of structures. Proceedings of the Third International Conference in Structural Safety and Reliability, ICOSSAR'81; 1981. p. 409-20.

[2] Hisada T, Nakagiri S. Role of stochastic finite element method in structural safety and reliability. Proceedings of the Fourth International Conference on Structural Safety and Reliability, ICOSSAR'85; 1985. p. 385-95.

[3] Liu W-K, Belytschko T, Mani A. Probabilistic finite elements for non linear structural dynamics. Comput Meth Appl Mech Engng 1986;56: $61-86$.

[4] Liu W-K, Belytschko T, Mani A. Random field finite elements. Int J Numer Meth Engng 1986;23(10):1831-45.

[5] Takada T. Weighted integral method in stochastic finite element analysis. Prob Engng Mech 1990;5(3):146-56.

[6] Deodatis G. The weighted integral method, I: stochastic stiffness matrix. J Engng Mech 1991;117(8):1851-64.

[7] Deodatis G, Shinozuka M. The weighted integral method, II: response variability and reliability. J Engng Mech 1991;117(8):1865-77.

[8] Ditlevsen O, Madsen H. Structural reliability methods. Chichester: Wiley; 1996.

[9] Ghanem R-G, Spanos P-D. Stochastic finite elements-a spectral approach. Berlin: Springer; 1991.

[10] Ghanem R-G, Brzkala V. Stochastic finite element analysis of randomly layered media. J Engng Mech 1996;122(4):361-9.

[11] Waubke H. Dynamische Berechnungen für den Halbraum mit streuenden Parametern mittels orthogonaler Polynome. PhD Thesis, Technische Universität, München; 1996.

[12] Ghanem R-G. Probabilistic characterization of transport in heterogeneous media. Comput Meth Appl Mech Engng 1998;158:199-220.

[13] Ghanem R-G. Stochastic finite elements with multiple random nonGaussian properties. J Engng Mech, ASCE 1999;125(1):26-40.

[14] Anders M, Hori M. Stochastic finite element method for elasto-plastic body. Int J Numer Meth Engng 1999;46(11):1897-916.

[15] Ghanem R-G, Spanos P-D. Spectral stochastic finite-element formulation for reliability analysis. J Engng Mech 1991;117(10): $2351-72$.

[16] Ghanem R-G, Ghiocel D. A comparative analysis of FORM/SORM and polynomial chaos expansion for highly nonlinear systems. In: Lin Y, Su T, editors, vol. 1. Proceedings of the 11th ASCE Specialty 
Conference on Engineering Mechanics, Fort Lauderdale, FL; 1996. p. $535-8$.

[17] Der Kiureghian A, Ke J-B. The stochastic finite element method in structural reliability. Prob Engng Mech 1988;3(2):83-91.

[18] Liu P-L, Der Kiureghian A. Finite element reliability of geometrically non linear uncertain structures. J Engng Mech, ASCE 1991;117(8): $1806-25$.

[19] Zhang Y, Der Kiureghian A. Finite element reliability methods for inelastic structures. Technical Report no UCB/SEMM-97/05, Department of Civil Engineering, University of California, Berkeley; 1997.

[20] Vanmarcke E-H, Grigoriu M. Stochastic finite element analysis of simple beams. J Engng Mech, ASCE 1983;109(5):1203-14.

[21] Li C-C, Der Kiureghian A. Optimal discretization of random fields. J Engng Mech 1993;119(6):1136-54.

[22] Zhang J, Ellingwood B. Orthogonal series expansions of random fields in reliability analysis. J Engng Mech, ASCE 1994;120(12): 2660-77.

[23] Ditlevsen O. Dimension reduction and discretization in stochastic problems by regression method. In: Casciati F, Roberts B, editors. Mathematical models for structural reliability analysis. CRC Mathematical Modelling Series; 1996. p. 51-138. Chapter 2.

[24] Sudret B, Der Kiureghian A. Stochastic finite elements and reliability: a state-of-the-art report. Technical Report no UCB/SEMM-2000/08, University of California, Berkeley; 2000. 173 p.

[25] Ghanem R-G, Kruger R. Numerical solution of spectral stochastic finite element systems. Comput Meth Appl Mech Engng 1996;129(3): 289-303.
[26] Zhang Y, Der Kiureghian A. Two improved algorithms for reliability analysis. In: Rackwitz R, Augusti G, Borr A, editors. Proceedings of the Sixth IFIP WG7.5 Reliability and Optimization of Stuctural Systems; 1995.

[27] Liu P-L, Der Kiureghian: A. Optimization algorithms for structural reliability. Struct Safety 1991;9:161-77.

[28] Abdo T, Rackwitz R. A new P-point algorithm for large time invariant and time-variant reliability problems. Reliability and optimization of structures. Proceedings of the Third WG 7.5 IFIP Conference, Berkeley; 1990.

[29] Zhang Y, Der Kiureghian A. Dynamic response sensitivity of inelastic structures. Comput Meth Appl Mech Engng 1993;108:23-36.

[30] Der Kiureghian A, Zhang Y. Space-variant finite element reliability analysis. Comput Meth Appl Mech Engng 1999;168:173-83.

[31] Der Kiureghian A. The geometry of random vibrations and solutions by form and sorm. Prob Engng Mech 2000;15(1):81-90.

[32] Melchers R-E. Structural reliability analysis and prediction. New York: Wiley; 1999.

[33] Haukaas T, Der Kiureghian A. A computer program for nonlinear finite element analysis. Proceedings of the Eighth International Conference on Structural Safety and Reliability, Newport Beach, CA (CD-ROM); 2001.

[34] Ghanem R-G. The nonlinear gaussian spectrum of log-normal stochastic processes and variables. J Appl Mech, ASME 1999;66: $964-73$. 